Pacific

Journal of

Mathematics

REPRESENTATIONS OF THE BRAID GROUP $B_{3}$ AND OF $S L(2, \mathrm{Z})$

ImRe Tuba AND HANS WenzL

Volume $197 \quad$ No. 2

February 2001 


\title{
REPRESENTATIONS OF THE BRAID GROUP $B_{3}$ AND OF $S L(2, \mathrm{Z})$
}

IMRE TUBA AND HANS WENZL

\begin{abstract}
We give a complete classification of simple representations of the braid group $B_{3}$ with dimension $\leq 5$. As an application of our techniques, we also obtain nontrivial $q$-versions of some of Deligne's formulas for dimensions of representations of exceptional Lie groups.
\end{abstract}

1.

We give a complete classification of all simple representations of $B_{3}$ for dimension $d \leq 5$ over an algebraically closed field $K$ of any characteristic. To describe our result in detail, recall that $B_{3}$ is given by generators $\sigma_{1}$ and $\sigma_{2}$ which satisfy the relation $\sigma_{1} \sigma_{2} \sigma_{1}=\sigma_{2} \sigma_{1} \sigma_{2}$. Moreover, it is well-known that the center of $B_{3}$ is generated by $\zeta=\left(\sigma_{1} \sigma_{2}\right)^{3}$. It is easy to see that $\zeta$ acts on a simple $d$-dimensional $B_{3}$ - module via the scalar $\delta$ which satisfies the equation $\delta^{d}=\operatorname{det}(A)^{6}$, where $A$ is the linear endomorphism via which $\sigma_{1}$ acts on $V$. Our main result states that the eigenvalues of $A$ and the scalar $\delta$ completely determine a simple representation of $B_{3}$ for dimension $\leq 5$, up to equivalence; for $d \leq 3$ it is uniquely determined by the eigenvalues of $A$. Moreover, such simple representations exist if and only if the eigenvalues do not belong to the zero set of certain polynomials in the eigenvalues and $\delta$ which we list explicitly (see Proposition 2.8, Section 2.10 and 2.11, Remark 4).

One of the motivations for this paper was studying braided tensor categories by analysing braid representations. The categories under consideration have a Grothendieck semiring isomorphic to the one of a semisimple Lie group. It turns out that in this context it suffices to classify representations of $B_{3}$ up to dimension 5. Indeed, as an application, we obtain uniform formulas for the categorical dimensions of objects in the second tensor power of the adjoint representation in braided tensor categories closely related to the conjectured series of exceptional Lie algebras, as proposed by Deligne and Vogel.

Our approach in this paper is quite elementary: We first show that assuming a certain triangular form of the matrices $A$ and $B$ of the generators of $B_{3}$, the braid relation reduces to checking the values of certain coefficients of the matrix $B A$. We then show that for dimension $d \leq 5$ one can 
always assume such a triangular form, and that the matrix coefficients are determined by the eigenvalues and $\delta$, up to certain renormalizations. These explicit representations are then used to compute polynomials which determine whether the representation is simple or not. Moreover, we show in the last section how these polynomials can be used to compute categorical dimensions of objects in braided categories, and we give explicit formulas for them.

After having completed this research we learned of other approaches towards classifying braid representations, notably via local systems by $\mathrm{N}$. Katz, and via quivers. These approaches as well as the case with $d \geq 6$ are discussed in the end of Chapter 2.

We would like to thank M. Hunziker, N. Wallach, A. Wassermann, B. Westbury and, in particular, P. Deligne for useful discussions.

1.1. Let $B_{3}$ be Artin's braid group, given by generators $\sigma_{1}$ and $\sigma_{2}$ and the relation $\sigma_{1} \sigma_{2} \sigma_{1}=\sigma_{2} \sigma_{1} \sigma_{2}$. It is well-known that $B_{3}$ maps surjectively onto $S L(2, \mathbf{Z})$, e.g. via the map

$$
\sigma_{1} \mapsto\left(\begin{array}{cc}
1 & 1 \\
0 & 1
\end{array}\right), \quad \sigma_{2} \mapsto\left(\begin{array}{cc}
1 & 0 \\
-1 & 1
\end{array}\right) .
$$

It is easy to check that this homomorphism maps

$$
\sigma_{1} \sigma_{2} \sigma_{1}=\sigma_{2} \sigma_{1} \sigma_{2} \mapsto S=\left(\begin{array}{cc}
0 & 1 \\
-1 & 0
\end{array}\right) \quad \text { and } \quad \sigma_{1} \sigma_{2} \mapsto U=\left(\begin{array}{cc}
1 & 1 \\
-1 & 0
\end{array}\right) \text {. }
$$

Moreover, the center of $S L(2, \mathbf{Z})$ is equal to \pm 1 , and the corresponding elements $\bar{S}$ and $\bar{U}$ in the quotient $P S L(2, \mathbf{Z})$ have orders 2 and 3 respectively. It is known that $P S L(2, \mathbf{Z})$ is isomorphic to the free product $\mathbf{Z}_{2} * \mathbf{Z}_{3}$ of a cyclic group of order 2 with a cyclic group of order 3 , and that the isomorphism can be chosen such that $\bar{S}$ and $\bar{U}$ are the generating elements of these cyclic groups. Finally, the center $Z$ of $B_{3}$ is generated by $\left(\sigma_{1} \sigma_{2}\right)^{3}$, and $B_{3} / Z \cong P S L(2, \mathbf{Z})$.

1.2. In the following we assume $A$ and $B$ to be invertible $d \times d$ matrices over the algebraically closed field $K$ satisfying $A B A=B A B$. Hence $\sigma_{1} \mapsto A$, $\sigma_{2} \mapsto B$ defines a representation of $B_{3}$. Let $\lambda_{1}, \ldots \lambda_{d}$ be the eigenvalues of $A$ (with $\lambda_{i}$ not necessarily distinct from $\lambda_{j}$ for $i \neq j$ ) and define $\delta=$ $\operatorname{det}(A)^{6 / d}=\left(\prod_{i=1}^{d} \lambda_{i}\right)^{6 / d}$.

Lemma. (a) Conjugation via $A B A$ maps $A$ to $B$ and $B$ to $A$.

(b) $A B A(A B)^{-1}=B$ and $B A B(B A)^{-1}=A$.

(c) $(A B A)^{2}=\delta 1$, and $(A B A)^{-1}=\delta^{-1} A B A$ if $A$ and $B$ generate the full $d \times d$ matrix ring. 
(d) For any choice $\delta^{1 / 6}$ of a sixth root of $\delta$ the map $\sigma_{1} \mapsto \delta^{-1 / 6} A, \sigma_{2} \mapsto$ $\delta^{-1 / 6} B$ defines a representation of $B_{3}$ and of $P S L(2, \mathbf{Z}) \cong B_{3} / Z$.

(e) If $\left\{e_{1}, e_{2}, \ldots, e_{d}\right\}$ is a basis of eigenvectors of $A$, and $b_{i}=A B A e_{i}$, then $\left\{b_{1}, b_{2}, \ldots, b_{d}\right\}$ is a basis of eigenvectors of $B$.

Proof. It follows from the braid relation that $B A B^{-1}=A^{-1} B A$, from which one easily deduces claims (a) and (b). For (c), observe that $\left(\sigma_{1} \sigma_{2} \sigma_{1}\right)^{2}$ $=\left(\sigma_{1} \sigma_{2}\right)^{3}$ is in the center of $B_{3}$. As we assume an irreducible representation of $B_{3}$, the center has to act via a scalar matrix, say $\lambda 1$. Comparing determinants (using $\operatorname{det}(A)=\operatorname{det}(B)$, we get $\lambda^{d}=\operatorname{det}(A)^{6}$. Statement (e) follows from (a); (d) is clear.

1.3. We say that the $d \times d$ matrices $A$ and $B$ are in ordered triangular form if $A$ is an upper triangular matrix with eigenvalue $\lambda_{i}$ as $i$-th diagonal entry, and $B$ is a lower triangular matrix with eigenvalue $\lambda_{d+1-i}$ as $i$-th diagonal entry.

Lemma. Assume $A$ and $B$ are in ordered triangular form, satisfying $A B A$ $=B A B$. Then:

(a) Any $d \times d$ matrix $F=\left(f_{i j}\right)$ such that $F B F^{-1}=A$ has matrix entries $f_{i j}=0$ for $i+j>d+1$. This holds, in particular, for $F=B A$.

(b) Any $d \times d$ matrix $E=\left(e_{i j}\right)$ such that $E A E^{-1}=B$ has matrix entries $e_{i j}=0$ for $i+j<d+1$. This holds, in particular, for $E=A B$.

Proof. Let $\left\{w_{1}, w_{2}, \ldots, w_{d}\right\}$ be the standard basis for $K^{d}$. By assumption, the eigenspace of $A$ corresponding to eigenvalues $\lambda_{1}, \ldots, \lambda_{r}$ is equal to $\operatorname{span}\left\{w_{1}, w_{2}, \ldots, w_{r}\right\}$, for $r=1,2, \ldots, d$, while the corresponding eigenspace for $B$ is equal to span $\left\{w_{\overline{1}}, w_{\overline{2}}, \ldots, w_{\bar{r}}\right\}$, where $\bar{i}=d+1-i$. Hence $F$ maps $\operatorname{span}\left\{w_{1}, w_{2}, \ldots, w_{r}\right\}$ onto $\operatorname{span}\left\{w_{\overline{1}}, w_{\overline{2}}, \ldots, w_{\bar{r}}\right\}$, from which we deduce the triangular shape of $F$. The second statement in (a) follows from Lemma 1.2(b). Statement (b) is proved similarly.

Proposition. Assume $A$ and $B$ are in ordered triangular form, satisfying $A B A=B A B$. Let $\bar{i}=d+1-i$. After rescaling our basis vectors, if necessary, we can assume:

(a) $A B A$ is a skew diagonal matrix, with $(A B A)_{i, \bar{i}}=(-1)^{i+1} \delta^{1 / 2}$.

(b) $b_{i j}=(-1)^{i+j} a_{\bar{i}, \bar{j}}$.

(c) $\sum_{k=1}^{i}(-1)^{k+i} a_{\bar{i}, \bar{k}} a_{k, \bar{i}}=(B A)_{i, \bar{i}}=(-1)^{i+1} \delta^{1 / 2} / \lambda_{i}$.

(d) If $i+j>d+1, \sum_{k=1}^{\min (i, j)}(-1)^{k+i} a_{\bar{i}, \bar{k}} a_{k, j}=(B A)_{i j}=0$.

(e) Statements (a)-(d) also hold for the coefficients of the matrices $A^{\prime}=$ $D A D^{-1}$ and $B^{\prime}=D B D^{-1}$ if $D$ is a diagonal matrix with $d_{i i}=d_{\overline{i i}} \neq 0$. In particular, if the entries $a_{i d}$ for $1<i \leq[(d+1) / 2]$ are nonzero, we can choose arbitrary nonzero numbers for them. 
Proof. Statements (a) and (b) follow from the previous Lemma and Lemma 1.2(a) and (c). Statements (c) and (d) follow from explicit matrix computations, exploiting the triangular shapes of $A, B$ and $B A$, and using statement (a). For (e), observe that conjugation by $D$ does not change $A B A$, and also leaves $A^{\prime}$ and $B^{\prime}$ in ordered triangular form. One can now prove statements (a)-(d) for $A^{\prime}$ and $B^{\prime}$ as we did before for $A$ and $B$.

Corollary. (a) $a_{1 d}=\delta^{1 / 2} / \lambda_{1} \lambda_{d}$.

(b) $a_{2, d-1}=\left(\lambda_{2} a_{d-1, d} a_{1, d-1}-\delta^{1 / 2}\right) / \lambda_{2} \lambda_{d-1}$.

Proof. By Proposition, (c), we have $\delta^{1 / 2} / \lambda_{1}=(B A)_{1 d}$, with the latter being equal to $a_{1 d} \lambda_{d}$. Statement (b) follows similarly, using Proposition (c).

Proposition 1.4. Let $A$ be an upper triangular matrix with eigenvalues $\lambda_{1}, \lambda_{2}, \ldots, \lambda_{d}$ down the diagonal, and let $S$ be a skew-diagonal matrix with $S^{2}=c 1$ for some constant $c$ such that $B=S A S^{-1}$. Moreover, assume:

(a) $(B A)_{i j}=0$ for $i+j>d+1$.

(b) $\lambda_{i}(B A)_{i \bar{i}}=c s_{i, \bar{i}}$.

Then $A$ and $B$ satisfy the braid relations.

Proof. Observe that $S^{-1}$ is a scalar multiple of $S$. Hence $B=S^{-1} A S=$ $S A S^{-1}$. We get from this $A B=S(B A) S^{-1}$, hence $(A B)_{i j}=0$ for $i+j<$ $d+1$. Exploiting the triangular shapes of the matrices, we get that $A(B A)$ is upper skew-triangular and $(A B) A$ is lower skew-triangular. Hence $A B A$ is of skew-diagonal shape, with $(A B A)_{i, \bar{i}}=\lambda_{i}(B A)_{i, \bar{i}}=c s_{i \bar{i}}$, and $A B A$ commutes with $S$. Hence

$$
B A B=S(A B A) S^{-1}=A B A .
$$

\subsection{An example.}

Let $V$ be a $(d+1)$-dimensional vector space, with a basis labeled by $0,1, \ldots, d$, and let $\lambda_{0}, \lambda_{1}, \ldots, \lambda_{d}$ be parameters satisfying $\lambda_{i} \lambda_{d-i}=c$ for a fixed constant $c$. Only for this subsection we define $\bar{i}=d-i$. Then we get a $\left[\frac{d+1}{2}\right]$ parameter family of representations of $B_{3}$ via the matrices

$$
A=\left(\left(\frac{\bar{i}}{\bar{j}}\right) \lambda_{j}\right)_{i j}, \quad B=\left((-1)^{i+j}\left(\begin{array}{l}
i \\
j
\end{array}\right) \lambda_{\bar{i}}\right)_{i j} .
$$

To prove this, it satisfies to check the conditions of Proposition 1.4, with $S$ being the skew-diagonal matrix defined by $s_{i j}=\left(\delta_{i, \bar{j}}(-1)^{i} \lambda_{\bar{i}}\right)$. This can be fairly easily checked, using the identity

$$
\sum_{k=0}^{d}(-1)^{i+k}\left(\begin{array}{l}
i \\
k
\end{array}\right)\left(\begin{array}{l}
\bar{k} \\
\bar{j}
\end{array}\right)=(-1)^{i}\left(\begin{array}{l}
d-i \\
d-j
\end{array}\right)=(-1)^{i}\left(\begin{array}{l}
\bar{i} \\
\bar{j}
\end{array}\right) .
$$

This identity is well-known, and can be easily proved by induction on $i$. 


\section{Representations of $B_{3}$ of dimension $d \leq 5$.}

2.1. In the following let $V$ be a $B_{3}$-module over the field $K$. Let $\left\{e_{1}, e_{2}, \ldots\right.$, $\left.e_{d}\right\}$ be a basis of generalized eigenvectors of $A$, and let $\left\{b_{1}, b_{2}, \ldots, b_{d}\right\}$ be a basis of generalized eigenvectors of $B$ with $b_{i}=A B A e_{i}$ for $i=1,2, \ldots, d$. We shall always assume that if $A$ and $B$ have Jordan blocks, the labeling is chosen such that they are upper triangular. As a consequence of our assumptions and Lemma 1.2, there exist scalars $c_{i j}, 1 \leq i, j \leq d$ such that

$$
b_{i}=\sum_{j=1}^{d} c_{j i} e_{j} \quad \text { and } \quad e_{i}=\delta^{-1} \sum_{j=1}^{d} c_{j i} b_{j} .
$$

As $(A B A)^{2}=\delta 1$, the matrix $A B A$ is diagonalizable. We will usually refer to an eigenvalue of $A B A$ just as $\delta^{1 / 2}$, not specifying which root we choose. We shall also consider the subspaces

$$
\begin{gathered}
W=\operatorname{span}\left\{e_{1}, e_{2}, \ldots, e_{d-1}\right\} \cap \operatorname{span}\left\{b_{1}, b_{2}, \ldots, b_{d-1}\right\} \\
W^{\prime}=\bigcap_{i \in \mathbf{N}}(A B)^{i}\left(\operatorname{span}\left\{e_{1}, e_{2}, \ldots, e_{d-1}\right\}\right) .
\end{gathered}
$$

Observe that $W^{\prime} \subset W$, as $A B e_{i}=A B A A^{-1} e_{i}=\lambda_{i}^{-1} b_{i}$. Moreover, $W$ is invariant under $A B A$ and $W^{\prime}$ is invariant under $A B$. Also observe that $W$ has codimension $\leq 2$ and $W^{\prime}$ has codimension $\leq 3$, being the intersection of 2 resp. 3 subspaces of $V$ with codimension 1 . We shall need the following simple observations:

(i) Each of the sets $\left\{\sigma_{1}, \sigma_{2}\right\},\left\{\sigma_{1}, \sigma_{1} \sigma_{2}\right\}$ or $\left\{\sigma_{1} \sigma_{2} \sigma_{1}, \sigma_{1} \sigma_{2}\right\}$ generates $B_{3}$.

(ii) Let $I$ be a subset of $\{1,2, \ldots, d\}$. If $V^{\prime}=\operatorname{span}\left\{e_{i}, i \in I\right\}=$ $\operatorname{span}\left\{b_{i}, i \in I\right\}$, then $V^{\prime}$ is a $B_{3}$-submodule.

(iii) If $W$ has codimension 1 , it would coincide with $\operatorname{span}\left\{e_{1}, e_{2}, \ldots, e_{d-1}\right\}$ and with $\operatorname{span}\left\{b_{1}, b_{2}, \ldots, b_{d-1}\right\}$, and therefore would be a $B_{3}$-submodule, by (ii).

(iv) If both $W$ and $W^{\prime}$ have codimension 2, they would coincide; this space would be invariant under both $A B A$ and $A B$, from which one easily deduces that it is a $B_{3}$-submodule, by (i).

Proposition 2.2. Let $V$ be a simple $B_{3}$-module of dimension $d \leq 5$. Then an eigenvector of $A$, say $e_{i}$ can not be contained in a proper subspace of $V$ which is invariant under $B$ and contains $b_{i}=A B A e_{i}$.

Proof. We choose the labeling of (generalized) eigenvectors so that $e_{1}$ is contained in a $B$-invariant subspace spanned by generalized eigenvectors $b_{1}$, $\ldots, b_{d-1}$. Let $W$ and $W^{\prime}$ be as in 2.1. By 2.1(iii) and (iv), the claim follows immediately unless $W$ has codimension 2 and $W^{\prime}$ has codimension 
3. As $W$ has at least dimension 1 , containing $e_{1}$, we get the claim for $d=2$ immediately.

$d=3$ : Here $W$ has to be 1-dimensional, containing both $e_{1}$ and $b_{1}$, i.e., it is an eigenspace of both $A$ and $B$.

$d=4$ : By assumption, $W$ contains both $e_{1}$ and $b_{1}$ and has dimension 2 . If $e_{1}$ and $b_{1}$ are linearly dependent, the claim follows from (2.1)(ii). Hence we can assume them to be a basis for $W$. As $\operatorname{dim} W^{\prime}=1$, there exists an eigenvector $v=\alpha e_{1}+\beta b_{1}$ of $A B$ in $W^{\prime}$. We can assume $\beta \neq 0$, as otherwise $v$ would be an eigenvector of both $A$ and $A B$, from which the claim would follow by 2.1(i). But then

$$
\mu\left(\alpha e_{1}+\beta b_{1}\right)=A B\left(\alpha e_{1}+\beta b_{1}\right)=\lambda_{1}^{-1} b_{1}+\beta \lambda_{1} A b_{1},
$$

where $\mu$ is the eigenvalue of $v$. We deduce from this $A b_{1} \in W$, and also $\delta B e_{1}=B A B A b_{1} \in B A B(W)=W$. Hence $W$ is invariant under both $A$ and $B$.

$d=5$ : We can assume $W$ to have a basis $\left\{e_{1}, b_{1}, x\right\}$, with $x$ an eigenvector of $A B A$ with eigenvalue $\delta^{1 / 2}$ (see 2.1). As $A B$ is diagonalizable, we can choose a basis of eigenvectors $v_{i}=\alpha^{(i)} e_{1}+\beta^{(i)} b_{1}+\gamma^{(i)} x, i=1,2$ for $W^{\prime}$. We have $A B v_{i}=w_{i}+\alpha^{(i)} \lambda_{1}^{-1} b_{1}$, with $w_{i}=\lambda_{1} \beta^{(i)} A b_{1}+\gamma^{(i)} A B x$, for $i=1,2$. As $A B v_{i}$ and $b_{1}$ are in $W$, so is $w_{i}$, for $i=1,2$.

Case 1: If $w_{1}$ and $w_{2}$ are linearly dependent, then there exist scalars $\nu_{1}$ and $\nu_{2}$ such that $\nu_{1} v_{1}+\nu_{2} v_{2} \neq 0$ and $\nu_{1} w_{1}+\nu_{2} w_{2}=0$. But then $A B\left(\nu_{1} v_{1}+\right.$ $\left.\nu_{2} v_{2}\right) \in W^{\prime}$ is a nonzero multiple of $b_{1}$; in particular, $b_{1} \in W^{\prime}$. But then also

$$
\delta \lambda_{1} e_{1}=\delta A e_{1}=A(B A B) b_{1}=(A B)^{2} b_{1} \in W^{\prime} .
$$

Hence $W^{\prime}$ is spanned by $e_{1}$ and $b_{1}$, and therefore is also invariant under $A B A$.

Case 2: If $w_{1}$ and $w_{2}$ are linearly independent, then also $A B x$ and $A b_{1}$ are in $W$. We conclude $B e_{1} \in W$ as in the case $d=4$. Moreover, also $\delta^{1 / 2} B^{-1} x=B^{-1} B A B x=A B x \in W$, i.e., $B^{-1} x$ is a linear combination of $e_{1}, b_{1}$ and $x$. If the coefficient of $x$ is not equal to 0 in this linear combination, we can multiply it by $B$ and solve for $B x$, which shows that it is in $W$. In this case, $W$ is invariant under both $B$ and $B A B$. If $B^{-1} x$ is a linear combination of only $e_{1}$ and $b_{1}$, we can multiply this linear combination by $A B$ to obtain for $A x$ a linear combination in $b_{1}$ and $A b_{1} \in W$. Hence $W$ is invariant under $A$ and $A B A$. The claim follows, using 2.1(i).

Corollary. Let $V$ be a simple $B_{3}$-module with dimension $d \leq 5$. Then the minimal polynomial of A coincides with its characteristic polynomial.

Proof. Let $b \in V$, let $\tilde{S}=\operatorname{span}\left\{A^{i} b, i=0,1, \ldots\right\}$, and let $\lambda$ be an eigenvalue of $A$. Then it is well-known that the intersection of the eigenspace of the eigenvalue $\lambda$ (for $A$ ) with $\tilde{S}$ has at most dimension 1 . To see this directly, let $p_{\lambda}$ be the projection onto the generalized eigenspace of $A$ for the eigenvalue 
$\lambda$, with the kernel being the direct sum of the generalized eigenspaces for the other eigenvalues. Then we get

$$
p_{\lambda} \tilde{S}=\operatorname{span}\left\{A^{i} p_{\lambda} b, i=0,1, \ldots\right\}=\operatorname{span}\left\{(A-\lambda)^{i} p_{\lambda} b, i=0,1, \ldots\right\} .
$$

The claim now follows from the fact that the nonzero elements in $\{(A-$ $\left.\lambda)^{i} p_{\lambda} b, i=0,1, \ldots\right\}$ are linearly independent, and $A$ acts as a full Jordan block on their span.

If the minimal polynomial of $A$ does not coincide with the characteristic polynomial, there exists an eigenvalue, say $\lambda_{1}$, whose eigenvectors span a subspace $E$ of dimension at least 2 . If there is an eigenvalue distinct from $\lambda_{1}$, say $\lambda_{j}$, pick an eigenvector $b_{j}$ of $B$ belonging to $\lambda_{j}$. Then $\operatorname{span}\left\{A^{s} b_{j}, s=\right.$ $0,1, \ldots, d-2\}$ forms an $A$-invariant subspace $S^{\prime}$ such that $\operatorname{dim} S^{\prime} \cap E \leq 1$, as proved in the last paragraph. Let $S$ be the subspace generated by $S^{\prime}$ and the eigenvectors of $A$ with eigenvalue $\lambda_{j}$. Then also $\operatorname{dim} S \cap E \leq 1$, i.e., $S$ is a proper $A$-invariant subspace of $V$ which contains both $b_{j}$ and $B A b_{j}$. Hence $V$ could not be a simple $B_{3}$-module.

If $A$ only has one eigenvalue, $\operatorname{dim} E \geq 2$ implies that we have at least 2 different Jordan blocks. Let $b_{j}$ be an eigenvector of $B$ belonging to a block of maximum length. Let $S^{\prime}=\operatorname{span}\left\{A^{s} b_{j}, s=0,1, \ldots, d-2\right\}$, which is a proper subspace of $V$ by assumption. If $S^{\prime} \operatorname{did}$ not contain $e_{j}=B A b_{j}$, it would not contain the whole Jordan block of $e_{j}$ either. Hence the space $S$ spanned by $S^{\prime}$ and $\left\{e_{j}\right\}$ is a proper $A$-invariant subspace containing both $e_{j}$ and $b_{j}$, and hence is a $B_{3}$-submodule. If $S^{\prime}$ does contain $e_{j}$, it would be a proper $B_{3}$-submodule by the same argument.

Lemma 2.3. Let $e_{1}$ and $e_{2}$ be either 2 eigenvectors of $A$ with eigenvalues $\lambda_{1}$ and $\lambda_{2}$, or let them be generalized eigenvectors with $A e_{1}=\lambda e_{1}$ and $A e_{2}=\lambda e_{2}+e_{1}$.

(a) Let $V^{\prime}=\operatorname{span}\left\{e_{1}, e_{2}, b_{1}, b_{2}\right\}$. If $\operatorname{dim} V^{\prime} \leq 3$, then there exists a nonzero $B_{3}$-invariant subspace of $V$ of dimension $\leq 3$.

(b) Let $d=5$ and let $V^{\prime \prime}=\operatorname{span}\left\{e_{1}, e_{2}, e_{3}, b_{1}, b_{2}\right\}$, with $e_{3}$ a generalized eigenvector belonging to $e_{1}$ or $e_{2}$, or an eigenvector. If $\operatorname{dim} V^{\prime \prime} \leq 4$, it contains a nonzero $B_{3}$-invariant subspace.

Proof. The claim in (a) follows from 2.1(ii) if $\operatorname{dim} V^{\prime}=2$. Hence we can assume $\operatorname{dim} V^{\prime}=3$. Let $W=\operatorname{span}\left\{e_{1}, e_{2}\right\} \cap \operatorname{span}\left\{b_{1}, b_{2}\right\}$. Then $\operatorname{dim} W=1$, as otherwise $\left\{e_{1}, e_{2}, b_{1}, b_{2}\right\}$ would be linearly independent. In particular, $W$ is spanned by an eigenvector $w=\alpha_{1} e_{1}+\alpha_{2} e_{2}$ of $A B A$. Let us assume first that both $e_{1}$ and $e_{2}$ are eigenvectors. If $\lambda_{1}=\lambda_{2}, w$ would be a common eigenvector of both $A$ and $A B A$, and we are done. Hence we can assume $\lambda_{1} \neq \lambda_{2}$. We can also assume $\alpha_{2} \neq 0$ in the expression for $w$, as otherwise $w$ would be an eigenvector of both $A$ and $A B A$. Moreover, we also have $\delta^{1 / 2} w=A B A w=\alpha_{1} b_{1}+\alpha_{2} b_{2}$. We now compute

$$
A B w=B^{-1} B A B\left(\alpha_{1} e_{1}+\alpha_{2} e_{2}\right)=\alpha_{1} \delta^{1 / 2} w+\alpha_{2}\left(\lambda_{2}^{-1}-\lambda_{1}^{-1}\right) b_{2},
$$




$$
(A B)^{2} w=\delta^{1 / 2} A\left(\alpha_{1} e_{1}+\alpha_{2} e_{2}\right)=\delta^{1 / 2}\left(\lambda_{1} w+\alpha_{2}\left(\lambda_{2}-\lambda_{1}\right) e_{2}\right) .
$$

The coefficients of $e_{2}$ and $b_{2}$ in the expressions above are nonzero. Hence the vectors $\left\{e_{2}, b_{2}, w\right\}$ and $\left\{w, A B w,(A B)^{2} w\right\}$ are bases for the same subspace. This proves that it is both invariant under $A B$ (second basis, as $(A B)^{3} w=$ $\delta w)$ and under $A B A$ (first basis). This proves statement (a) if both $e_{1}$ and $e_{2}$ are eigenvectors. If $e_{2}$ is a generalized eigenvector, we compute

$$
A B w=\delta^{1 / 2} \lambda^{-1} w-\delta^{1 / 2} \alpha_{2} \lambda^{-2} b_{1}, \quad(A B)^{2} w=\delta^{1 / 2} \lambda w+\delta^{1 / 2} \alpha_{2} e_{1} .
$$

One shows as before that $\operatorname{span}\left\{w, A B w,(A B)^{2} w\right\}=\operatorname{span}\left\{e_{1}, b_{1}, w\right\}$, from which one deduces the claim.

Observe that $V^{\prime} \subset V^{\prime \prime}$. Statement (b) follows immediately from (a) if $\operatorname{dim} V^{\prime}<4$. Hence we can assume $\operatorname{dim} V^{\prime}=4$ and $V^{\prime}=V^{\prime \prime}$, which therefore is invariant under $A B A$. Moreover, the space $U=\operatorname{span}\left\{e_{1}, e_{2}, e_{3}\right\} \cap$ $\operatorname{span}\left\{b_{1}, b_{2}, b_{3}\right\}$ has at least dimension 2 , being the intersection of 2 subspaces of $V^{\prime}$ with codimension 1 ; we can assume $\operatorname{dim} U=2$ as otherwise $U$ would be an invariant subspace. Hence it contains 2 linearly independent eigenvectors $w_{1}$ and $w_{2}$ of $A B A$. Moreover, if $w_{1}=\sum \alpha_{j} e_{j}$, we can assume $\alpha_{3} \neq 0$; otherwise $w_{1} \in \operatorname{span}\left\{e_{1}, e_{2}\right\} \cap \operatorname{span}\left\{b_{1}, b_{2}\right\}$ which is 0 (the vectors $e_{1}, e_{2}, b_{1}, b_{2}$ are a basis for $\left.V^{\prime}\right)$. Now the equality $A B A w_{1}=\delta^{1 / 2} w_{1}$ implies

$$
B A w=B\left(\sum_{j=1}^{3} \alpha_{j} \lambda_{j} e_{j}\right)=\sum_{j=1}^{3} \delta^{1 / 2} \alpha_{j} \lambda_{j}^{-1} e_{j}=\delta^{1 / 2} A^{-1} w .
$$

We want to show that we can assume $e_{3} \notin U$. If $e_{3}$ is a generalized eigenvector belonging to $e_{1}$ with $e_{3} \in U$, we can replace it by $e_{3}+\tau e_{1}$ for any scalar $\tau \in K$. This new vector would only be in $U$ for any choice of $\tau$ if $e_{1} \in U$. But then $U$ would be spanned by $e_{1}$ and $e_{3}$, and would be a $B_{3^{-}}$ submodule, being invariant under $A$ and $A B A$. If $e_{3}$ is an eigenvector in $U \subset \operatorname{span}\left\{b_{1}, b_{2}, b_{3}\right\}$, the claim follows from Prop. 2.2.

So we can assume that $X=\operatorname{span}\left\{e_{3}, w_{1}, w_{2}\right\}$ coincides with $\operatorname{span}\left\{e_{1}, e_{2}\right.$, $\left.e_{3}\right\}$. If $e_{4}$ and $e_{5}$ were eigenvectors with same eigenvalue $\lambda$, then either $A B A e_{3} \in X$ (which would make $X$ invariant under both $A$ and $A B A$ ), or $\operatorname{span}\left(\left\{A B A e_{3}\right\} \cup X\right)$ would be equal to $\operatorname{span}\left(\left\{\tilde{e}_{4}\right\} \cup X\right)$, where $\tilde{e}_{4}$ is a linear combination of $e_{4}$ and $e_{5}$, and, in particular, it is an eigenvector of $A$. Using these 2 different spanning sets, we see that we would obtain a 4-dimensional subspace invariant under both $A$ and $A B A$.

Hence we can assume that $V / X$ has at most $2 A$-invariant 1-dimensional subspaces, and, similarly $V / \operatorname{span}\left\{b_{1}, b_{2}, b_{3}\right\}$ has at most $2 B$-invariant 1 dimensional subspaces. Expanding the vectors in the equation $(*)$ as a linear combination of $\left\{e_{3}, w_{1}, w_{2}\right\}$, and observing that $w_{1}, w_{2} \in \operatorname{span}\left\{b_{1}, b_{2}, b_{3}\right\}$, we obtain that $B e_{3}$ is congruent to a multiple of $e_{3}$ modulo $\operatorname{span}\left\{b_{1}, b_{2}, b_{3}\right\}$. But this would imply that $e_{3}$ together with $b_{1}, b_{2}, b_{3}$ spans a $B$-invariant subspace of dimension $\leq 4$. Statement (b) now follows from Proposition 
2.2, if $e_{3}$ is an eigenvector. If $e_{3}$ is a generalized eigenvector belonging to, say $e_{1}$, we could show as before that also $e_{3}^{\prime}=e_{3}+\tau e_{1}$ is in a 4-dimensional $B$-invariant subspace containing $b_{1}, b_{2}$ and $b_{3}$. As this is true for any $\tau \in K$, and there are only at most 2 such subspaces, we obtain that also $e_{1}$ itself is in a $B$-invariant subspace containing $b_{1}$. The claim follows from Proposition 2.2 .

Proposition 2.4. Let $V$ be a simple $B_{3}$-module with dimension $\leq 5$. Then there exists a basis of $V$ with respect to which $A$ and $B$ act in ordered triangular form (see 1.3). Moreover, this is possible for any labeling of the generalized eigenvectors as long as $A$ appears in upper triangular Jordan form.

Proof. To construct such a basis, we can always assume at least one eigenvector $e_{1}$ for $A$ and $b_{1}$ for $B$, and at least one more (generalized) eigenvector $e_{2}$ as in Lemma 2.3. We define $w_{1}=e_{1}$ and $w_{d}=b_{1}$. For $d \geq 3$, observe that $c_{d 1} \neq 0$ by Proposition 2.2 , with $c_{i j}$ as in 1.3 , for $1 \leq i, j \leq d$. Hence we can define $w_{2}=e_{2}-\left(c_{d 2} / c_{d 1}\right) e_{1}$, which is both in $\operatorname{span}\left\{e_{1}, e_{2}\right\}$ and in $\operatorname{span}\left\{b_{1}, \ldots, b_{d-1}\right\}$. For $d \geq 4$, define $w_{d-1}=A B A w_{2}$, which is in $\operatorname{span}\left\{b_{1}, b_{2}\right\}$ and in $\operatorname{span}\left\{e_{1}, \ldots, e_{d-1}\right\}$. Finally, if $d=5$, we can express $b_{3}$ as a linear combination of $\left\{e_{1}, e_{2}, e_{3}, b_{1}, b_{2}\right\}$, by Lemma 2.3. Hence there exist scalars $\alpha_{1}$ and $\alpha_{2}$ such that $w_{3}=b_{3}-\alpha_{1} b_{1}-\alpha_{2} b_{2}$ is in $\operatorname{span}\left\{e_{1}, e_{2}, e_{3}\right\} \cap$ $\operatorname{span}\left\{b_{1}, b_{2}, b_{3}\right\}$. By construction, it follows that

(*) $\quad w_{i} \in \operatorname{span}\left\{e_{1}, e_{2}, \ldots, e_{i}\right\} \cap \operatorname{span}\left\{b_{1}, b_{2}, \ldots, b_{d+1-i}\right\} \quad$ for $1 \leq i \leq 3$.

It follows from $(*)$ that $\operatorname{span}\left\{w_{1}, w_{2}, \ldots, w_{i}\right\} \subset \operatorname{span}\left\{e_{1}, e_{2}, \ldots, e_{i}\right\}$ and that $\operatorname{span}\left\{w_{d+1-i}, w_{d+2-i}, \ldots, w_{d}\right\} \subset \operatorname{span}\left\{b_{1}, b_{2}, \ldots, b_{i}\right\}$. Let us check equality for $d=5$. This follows by construction for $i \leq 3$. But then $\operatorname{span}\left\{w_{1}, w_{2}, w_{3}\right\}$ $=\operatorname{span}\left\{e_{1}, e_{2}, e_{3}\right\}$ and $\operatorname{span}\left\{w_{4}, w_{5}\right\}=\operatorname{span}\left\{b_{1}, b_{2}\right\}$. The linear independence of the $w_{i}$ 's now follows from Lemma 2.3(b). Hence the inclusions below $(*)$ actually are equalities, from which easily deduces the triangular forms of $A$ and $B$ by induction on $i$. The cases $d<5$ are similar and easier to check.

Proposition 2.5. Let $V$ be a simple $B_{3}$-module of dimension $d$ with $d=$ 2,3 . Then there exists a basis for $V$ for which $A$ and $B$ acts via the matrices

$$
\begin{gathered}
A=\left(\begin{array}{cc}
\lambda_{1} & \lambda_{1} \\
0 & \lambda_{2}
\end{array}\right), \quad B=\left(\begin{array}{cc}
\lambda_{2} & 0 \\
-\lambda_{2} & \lambda_{1}
\end{array}\right) \quad \text { for } d=2 \\
A=\left(\begin{array}{ccc}
\lambda_{1} & \lambda_{1} \lambda_{3} \lambda_{2}^{-1}+\lambda_{2} & \lambda_{2} \\
0 & \lambda_{2} & \lambda_{2} \\
0 & 0 & \lambda_{3}
\end{array}\right), \quad B=\left(\begin{array}{ccc}
\lambda_{3} & 0 & 0 \\
-\lambda_{2} & \lambda_{2} & 0 \\
\lambda_{2} & -\lambda_{1} \lambda_{3} \lambda_{2}^{-1}-\lambda_{2} & \lambda_{1}
\end{array}\right) \\
\text { for } d=3 .
\end{gathered}
$$


Proof. For $d=2$ : By Proposition 2.4, we can assume $A$ and $B$ in ordered triangular form, with only the nonzero off-diagonal entries to be computed. Rescaling one of the basis vectors, we can assume $a_{12}=\lambda_{1}$. We obtain $b_{21}=-\lambda_{2}$ from the braid relation $A B A=B A B$.

For $d=3$ : Again, by Proposition 2.4, we only need to compute the nonzero off-diagonal entries of $A$ and $B$, which are in ordered triangular form. By Corollary 1.3, we can assume $a_{13}=\lambda_{2}=b_{31}$. If $a_{23}=0$, then also $b_{21}$, by Proposition 1.3(b). But then $\operatorname{span}\left\{w_{1}, w_{3}\right\}$ would be a subspace invariant under both $A$ and $B$, contradicting $V$ being simple. Hence we can assume $a_{23}=\lambda_{2}=-b_{21}$, by Prop. 1.3(e). Finally, we compute from $(B A)_{33}=0$ that $b_{32}=-\lambda_{1} \lambda_{3} \lambda_{2}^{-1}-\lambda_{2}$.

Proposition 2.6. Let $V$ be a simple 4-dimensional $B_{3}$-module, and let $D=$ $\sqrt{\lambda_{2} \lambda_{3} / \lambda_{1} \lambda_{4}}$. Then we can find a basis for $V$ with respect to which we get the matrices

$$
\begin{aligned}
A & =\left(\begin{array}{cccc}
\lambda_{1} & \left(1+D^{-1}+D^{-2}\right) \lambda_{2} & \left(1+D^{-1}+D^{-2}\right) \lambda_{3} & \lambda_{4} \\
0 & \lambda_{2} & \left(1+D^{-1}\right) \lambda_{3} & \lambda_{4} \\
0 & 0 & \lambda_{3} & \lambda_{4} \\
0 & 0 & 0 & \lambda_{4}
\end{array}\right) \\
B & =\left(\begin{array}{cccc}
\lambda_{4} & 0 & 0 & 0 \\
-\lambda_{3} & \lambda_{3} & 0 & 0 \\
D \lambda_{2} & -(D+1) \lambda_{2} & \lambda_{2} & 0 \\
-D^{3} \lambda_{1} & \left(D^{3}+D^{2}+D\right) \lambda_{1} & -\left(D^{2}+D+1\right) \lambda_{1} & \lambda_{1}
\end{array}\right) .
\end{aligned}
$$

Proof. For the proof, we first assume $A$ and $B$ in the form of Proposition 1.3. Then we get from its corollary

$$
\begin{gathered}
a_{14}=\delta^{1 / 2} / \lambda_{1} \lambda_{4}=\left(\lambda_{1} \lambda_{2} \lambda_{3} \lambda_{4}\right)^{1 / 4} D, \quad a_{23}=\left(a_{34} a_{13} \lambda_{2}-\delta^{1 / 2}\right) / \lambda_{2} \lambda_{3}, \\
a_{34}=\frac{\lambda_{3}}{a_{14}} a_{24}=\frac{\lambda_{1} \lambda_{3} \lambda_{4}}{\left(\lambda_{1} \lambda_{2} \lambda_{3} \lambda_{4}\right)^{3 / 4}} a_{24}, \quad a_{13}=\frac{\left(\lambda_{1} \lambda_{2} \lambda_{3} \lambda_{4}\right)^{3 / 4}}{\lambda_{1} \lambda_{2} \lambda_{4}} a_{12},
\end{gathered}
$$

where the last 2 equalities follow from $(B A)_{24}=0$ and $(B A)_{42}=0$. It follows from the equations above that $a_{34}=0$ if and only if $a_{24}=0$, and in this case, also $b_{21}=0=b_{31}$, by Prop. 1.3(b). But then $\operatorname{span}\left\{w_{2}, w_{3}\right\}$ would be a $B_{3}$-submodule, contradicting simplicity of $V$. By Proposition 1.3,(e) we can choose $a_{24}=\lambda_{4}$, from which one deduces

$$
a_{34}=\frac{\lambda_{1} \lambda_{3} \lambda_{4}^{2}}{\left(\lambda_{1} \lambda_{2} \lambda_{3} \lambda_{4}\right)^{3 / 4}} .
$$

We get from $(B A)_{34}=0$, using the substitution in Prop. 1.3(b) that

$$
a_{23}=\left(\lambda_{1} \lambda_{2} \lambda_{3} \lambda_{4}\right)^{1 / 4}(D+1) .
$$


Similarly, we obtain from $(B A)_{44}=0$, using Prop. 1.3(b) and the results so far

$$
a_{13}=\lambda_{4}^{-1} \lambda_{2} \lambda_{3}\left(D+1+D^{-1}\right), \quad a_{12}=\frac{\lambda_{1} \lambda_{2}^{2} \lambda_{3}}{\left(\lambda_{1} \lambda_{2} \lambda_{3} \lambda_{4}\right)^{3 / 4}}\left(D+1+D^{-1}\right) .
$$

To get $A$ and $B$ into the form as stated, it suffices to conjugate the matrices via the diagonal matrix $\operatorname{diag}\left(\lambda_{4} / a_{i 4}\right)_{i}$. This shows that there exist at most 2 representations of $B_{3}$ with prescribed eigenvalues $\lambda_{i}$ for $A$, up to conjugation, depending on the choice of the square root in the expression for $D$.

Observe that we used the equations $(B A)_{i j}=0$ for $j=4$ and $i>1$ for the computation of matrix entries above. It is easy to check $(B A)_{i j}=0$ for the remaining entries for which $i+j>5$. Hence condition (a) of Proposition 1.4 is satisfied, and condition (b) follows from Prop 1.3(d). Hence $A$ and $B$ satisfy the braid relations.

Proposition 2.7. Let $V$ be a simple 5-dimensional $B_{3}$-module. Then there exists a basis with respect to which $A$ and $B$ act as ordered triangular matrices. Moreover, the module is uniquely determined by the eigenvalues of $A$ and the choice $\gamma$ of $a 5^{\text {th }}$ root of $\operatorname{det}(A)$, up to equivalence.

Proof. We proceed as in the proof of Proposition 2.5, using the results of Section 1.3. By Corollary 1.3(a), we get

$$
a_{15}=\frac{\delta^{1 / 2}}{\lambda_{1} \lambda_{5}}=\frac{\gamma^{3}}{\lambda_{1} \lambda_{5}}
$$

If $a_{35}=0$, we can conclude from $(B A)_{35}=0$ that $a_{34} a_{25}=a_{35}\left(\lambda_{3}+a_{15}\right)=$ 0 . If $a_{34}=0$, then also $b_{31}=0=b_{32}$ by Prop. 1.3(b). In this case, $\operatorname{span}\left\{w_{1}, w_{2}, w_{4}, w_{5}\right\}$ is a $B_{3}$-submodule. Hence we can assume $a_{34} \neq 0$ and $a_{25}=0$. But then $a_{45}=\left(\lambda_{4} / a_{15}\right) a_{25}=0$ too by Corollary 1.3(b); and the matrix entries $b_{21}, b_{31}$ and $b_{41}$ are also equal to 0 , by Prop. 1.3(b). Hence $\operatorname{span}\left\{w_{1}, w_{5}\right\}$ is an invariant subspace.

So we can assume $a_{35} \neq 0$. Assume $a_{45}=0$; then also $a_{25}=\left(a_{15} / \lambda_{4}\right) a_{45}=$ 0 . We get from $(B A)_{45}=0$ that $a_{35} a_{23}=\lambda_{2} a_{45}+a_{24} a_{25}-a_{25} a_{15}=0$. Since $a_{35} \neq 0, a_{23}=0$. It follows that $b_{21}, b_{41}$ and $b_{43}$ are all equal to 0 , by Prop. $1.3(\mathrm{~b})$. This would entail that $\operatorname{span}\left\{w_{1}, w_{3}, w_{5}\right\}$ is a $B_{3}$-submodule.

Hence we can assume that $a_{i 5} \neq 0$ for $i=1,2, \ldots 5$. We can choose $a_{45}=\lambda_{4}$ and $a_{35}=a_{15}$, by Prop. 1.3(e). We now show that the equations in (1.3) completely determine the other entries of $A$ and $B$. As this does not seem to be completely straightforward, we include the details for the interested reader. Using $(B A)_{25}=0$ and $(B A)_{35}=0$ we get

$$
a_{25}=a_{15}=\frac{\gamma^{3}}{\lambda_{1} \lambda_{5}}, \quad a_{34}=a_{15}+\lambda_{3}=\frac{\gamma^{3}}{\lambda_{1} \lambda_{5}}+\lambda_{3} .
$$


From $(B A)_{24}=-\frac{\delta^{1 / 2}}{\lambda_{2}}=-\frac{\gamma^{3}}{\lambda_{2}}$,

$$
a_{24}=a_{14}-\frac{\gamma^{3}}{\lambda_{2} \lambda_{4}}
$$

Substituting this into $(B A)_{34}=0$, we find

$$
a_{14}=\frac{a_{34}\left(\lambda_{2} \lambda_{3} \lambda_{4}+\gamma^{3}\right)}{\left(a_{34}-a_{35}\right) \lambda_{2} \lambda_{4}}=\left(\frac{\lambda_{2} \lambda_{4}}{\gamma^{2}}+1\right)\left(\lambda_{3}+\frac{\gamma^{3}}{\lambda_{2} \lambda_{4}}\right),
$$

and hence

$$
a_{24}=\frac{\gamma^{3}}{\lambda_{1} \lambda_{5}}+\lambda_{3}+\gamma
$$

Using $(B A)_{52}=0$ we obtain

$$
a_{12}=\frac{\lambda_{2} a_{14}}{a_{15}}=\left(1+\frac{\gamma^{2}}{\lambda_{2} \lambda_{4}}\right)\left(\lambda_{2}+\frac{\gamma^{3}}{\lambda_{3} \lambda_{4}}\right) .
$$

Now $(B A)_{45}=0$ gives us

$$
a_{23}=a_{24}-a_{15}+\frac{\lambda_{2} \lambda_{4}}{a_{15}}=\gamma+\lambda_{3}+\frac{\gamma^{2}}{\lambda_{3}} .
$$

Finally, using $(B A)_{55}=0$, we get

$$
a_{13}=\left(\frac{\gamma^{2}}{\lambda_{3}}+\lambda_{3}+\gamma\right)\left(1+\frac{\lambda_{1} \lambda_{5}}{\gamma^{2}}\right) \text {. }
$$

This shows that all entries of $A$ and $B$ are uniquely determined by the eigenvalues $\lambda_{i}$ of $A$ and by a choice of a $5^{\text {th }} \operatorname{root}$ of $\operatorname{det}(A)$. It is now a straightforward computation to check the remaining conditions of Prop. 1.4 to prove that the matrices $A$ and $B$ do indeed define a representation of $B_{3}$. Alternatively, the existence question might be more easily settled using some of the methods discussed in Section 2.11.

2.8. Define for $1 \leq r \leq d$ the polynomials $P_{r}^{(d)}(x)=\prod_{i \neq r}\left(x-\lambda_{i}\right)$, where $1 \leq i \leq d$ with $i \neq r$. Observe that these polynomials divide the characteristic polynomial of $A$. It follows from the corollary of Prop. 2.2 that $P_{r}^{(d)}(A)$ is a nonzero rank 1 matrix; if the $d$ eigenvalues of $A$ are mutually distinct, it is a multiple of the projection onto the eigenspace of $\lambda_{r}$, with kernel being the direct sum of the eigenspaces of the other eigenvalues.

Now observe that for our braid representations, $A$ and $B$ are matrices with coefficients in the ring $R$ of Laurent polynomials in the $\lambda_{i}$ 's and $\gamma$, a $d$-th $\operatorname{root}$ of $\operatorname{det}(A)$ for $d=4,5$. As also the matrix $P_{r}^{(d)}(A) P_{s}^{(d)}(A) P_{r}^{(d)}(A)$ is a multiple of $P_{r}^{(d)}$, we obtain Laurent polynomials $Q_{r s}^{(d)}$ in $R$ by

$$
P_{r}^{(d)}(A) P_{s}^{(d)}(B) P_{r}^{(d)}(A)=Q_{r s}^{(d)} P_{r}^{(d)}(A) .
$$


Proposition. (a) $P_{1}^{(d)}(B) P_{d}^{(d)}(A)=Q_{1 d}^{(d)} E_{d d}$, where $E_{d d}$ is the matrix with $a 1$ in the $(d d)$-entry and zeroes everywhere else.

(b) If $Q_{i j}^{(d)}=0$, then $P_{i}^{(d)}(B) P_{j}^{(d)}(A)=0$.

(c) The matrix $\left(\prod_{r=i}^{d}\left(A-\lambda_{r}\right)\right)$ has nonzero entries in its last column at most in the 1-st until $i-1$-st row.

(d) The Laurent polynomials $Q_{r s}^{(d)}$ are given by

$$
\begin{gathered}
Q_{r s}^{(2)}=-\lambda_{r}^{2}+\lambda_{r} \lambda_{s}-\lambda_{s}^{2}, \quad Q_{r s}^{(3)}=\left(\lambda_{r}^{2}+\lambda_{s} \lambda_{k}\right)\left(\lambda_{s}^{2}+\lambda_{r} \lambda_{k}\right), \\
Q_{r s}^{(4)}=-\gamma^{-2}\left(\lambda_{r}^{2}+\gamma^{2}\right)\left(\lambda_{s}^{2}+\gamma^{2}\right)\left(\gamma^{2}+\lambda_{r} \lambda_{k}+\lambda_{s} \lambda_{l}\right)\left(\gamma^{2}+\lambda_{r} \lambda_{l}+\lambda_{s} \lambda_{k}\right), \\
\text { where }\{r, s, k, l\}=\{1,2,3,4\} . \\
Q_{r s}^{(5)}=\gamma^{-8}\left(\gamma^{2}+\lambda_{r} \gamma+\lambda_{r}^{2}\right)\left(\gamma^{2}+\lambda_{s} \gamma+\lambda_{s}^{2}\right) \prod_{k \neq r, s}\left(\gamma^{2}+\lambda_{r} \lambda_{k}\right)\left(\gamma^{2}+\lambda_{s} \lambda_{k}\right) .
\end{gathered}
$$

Proof. The statements are shown by straightforward computations. We give some details for the interested reader. For statement (a), observe that $P_{1}^{(d)}(B)$ is nonzero only in the last row, where it coincides with the righteigenvector of $B$ for the eigenvalue $\lambda_{1}$, and that $P_{d}^{(d)}(A)$ is nonzero only in the last column, where it coincides with the left eigenvector of $A$ for the eigenvalue $\lambda_{d}$. Using the triangular form of the matrices, and the fact that the nonzero diagonal entries are $P_{1}^{(d)}\left(\lambda_{1}\right)$ and $P_{d}^{(d)}\left(\lambda_{d}\right)$, respectively, these matrices can be computed easily. It is also obvious that $P_{1}^{(d)}(B) P_{d}^{(d)}(A)$ is a multiple of $E_{d d}$, and, multiplying it by $P_{d}^{(d)}(A)$ from the left, that this multiple is equal to $Q_{1 d}^{(d)}$.

Statement (b) follows from (a) and the fact that we obtain matrices in ordered triangular form independent of the labeling, Prop. 2.4. Statement (d) follows similarly from (a). Statement (c) is straightforward.

Main Theorem 2.9. Let $K$ be an algebraically closed field.

(a) Any simple $B_{3}$ module is uniquely determined by the eigenvalues of $A$, up to a choice of a square root $\gamma^{2}$ of $\operatorname{det}(A)($ for $d=4)$ resp. a 5-th root $\gamma$ of $\operatorname{det}(A)($ for $d=5)$.

(b) There exists a simple $B_{3}$ module $V$ of $K$-dimension $d \leq 5$ if and only if the eigenvalues $\lambda_{i}$ of $A$ and the quantities $\gamma^{2}$ (for $\left.d=4\right)$ and $\gamma($ for $d=5)$, as defined in (a), satisfy $Q_{r s}^{(d)} \neq 0$ for $r \neq s$ and $1 \leq r, s \leq d$, with $Q_{r s}^{(d)}$ as defined in 2.8. The eigenvalues $\lambda_{i}$ need not be mutually distinct for this statement.

Proof. Let $V$ be a simple $B_{3}$-module. By Propositions 2.5-7, we can assume a basis for $V$ such that $A$ and $B$ act via matrices as described there. This shows part (a). 
It remains to be shown for which values of the parameters the representations given there are simple. Let us assume $Q_{i j}^{(d)}=0$ for some $i \neq j$, $1 \leq i, j \leq d$. If $V$ is simple, we can assume $P_{i}^{(d)}(A) \neq 0$, by the corollary of Prop. 2.2. Hence there exists a vector $v$ for which $e_{i}=P_{i}^{(d)}(A) v$ is an eigenvector of $A$. As $Q_{j i}^{(d)}=0$, also $P_{j}^{(d)}(B) P_{i}^{(d)}(A)=0$, by Prop. 2.8(b). Obviously, $e_{i}$ is in the subspace spanned by $\left\{B^{r} e_{i}, 0 \leq r \leq d-2\right\} \cup\left\{b_{i}\right\}$, which is $B$-invariant; it is a proper subspace of $V$ as the minimal polynomial of the restriction of $B$ to it divides $P_{j}^{(d)}$. We obtain the existence of a nontrivial $B_{3}$-submodule from Prop. 2.2, i.e., $V$ can not be simple.

On the other hand, assume that $Q_{i j}^{(d)} \neq 0$ for all $1 \leq i \neq j \leq d$. Let $W$ be a nonzero $B_{3}$-submodule of $V$. Then it contains at least one eigenvector of $A$, say $e_{i}$. As $P_{i}^{(d)}(A) \neq 0$ by Prop. 2.8(a), we can assume this eigenvector to be of the form $e_{i}=P_{i}^{(d)}(A) v$ for some $v \in V$, also if $A$ is not diagonalizable. Let $j \neq i$. Then $=A B A P_{j}^{(d)}(B) P_{i}^{(d)}(A) v$ is an eigenvector of $A$ with eigenvalue $\lambda_{j}$, provided it is nonzero. Using $(A B A)^{-1} P_{i}(B) A B A=P_{i}(A)$, one easily checks that $P_{i}^{(d)}(B) e_{j}=Q_{i j}^{(d)}(A B A) e_{i}$, which is nonzero by our assumptions. Hence $W$ contains eigenvectors for each eigenvalue of $A$.

Case 1: Assume $A$ has at least 2 distinct eigenvalues, which we label $\lambda_{1}$ and $\lambda_{2}$. By triangularity of $A$, their eigenvectors can be chosen in the form $(1,0, \ldots)^{t}$ and $(*, 1,0, \ldots)^{t}$, which are both in $W$. It is easy to see from this that $e_{1}, e_{2}, b_{1}=A B A e_{1}$ and $b_{2}=A B A e_{2}$ together with $A b_{1}$ (which is the last column of $A$ ) span $V$, which therefore is equal to $W$.

Case 2: Assume $A$ and $B$ has only one eigenvalue, say $\lambda$. Then

$$
P_{1}^{(d)}(A) P_{d}^{(d)}(B)=(A-\lambda)^{d-1}(B-\lambda)^{d-1}=Q_{1 d} E_{d d} .
$$

As $Q_{1 d} \neq 0$, the eigenspaces of $\lambda$ for both $A$ and $B$ are 1-dimensional. If $W$ is a nonzero $B_{3}$-submodule, it therefore must contain the eigenvector $w_{d}$ of $B$. The set of vectors $S=\left\{(A-\lambda)^{i} w_{d}, i=0,1, \ldots d-1\right\}$ is in $W$. As $(A-\lambda)^{d-1} \neq 0$, so is its last column $(A-\lambda)^{d-1} w_{d}$, by triangularity of $A$. Hence $S$ is linearly independent. This finishes the proof.

Corollary. The simple $S L(2, \mathbf{Z})$ modules and the simple $P S L(2, \mathbf{Z})$ modules are given by all simple $B_{3}$-modules for which $\delta^{2}=1($ for $S L(2, \mathbf{Z}))$ and for which $\delta=1($ for $\operatorname{PSL}(2, \mathbf{Z}))$. Observe that $\delta=-\left(\lambda_{1} \lambda_{2}\right)^{3}$ for $d=2$ and $\delta=\left(\lambda_{1} \lambda_{2} \lambda_{3}\right)^{2}$ for $d=3$.

2.10. Parameter spaces for $B_{3}$ and $P S L(2, \mathbf{Z})$. Let $I_{d}$ be the ideal in $K\left[\lambda_{1}, \ldots, \lambda_{d}\right]$ (for $d \leq 3$ ) and in $K\left[\lambda_{1}, \ldots, \lambda_{d}, \gamma\right]$ (for $d=4,5$ ) generated by the least common multiple of the $Q_{r s}^{(d)}, 1 \leq r<s \leq d$. Let $N_{d}$ be the zero set of $I_{d}$ in $K^{d}$ (resp. in $K^{d+1}$ for $d>3$ ). Observe that $N_{d}$ is invariant under 
the action of the symmetric group, acting via permuting the coordinates $\lambda_{i}, 1 \leq i \leq 5$ in $K^{d}$. The main theorem can now be reformulated as follows:

Main Theorem'. There exists a 1-1 correspondence between equivalence classes of simple $B_{3}$ modules of dimension $d \leq 5$ and the $S_{d}$ orbits in $K^{d} \backslash N_{d}$.

Remarks 2.11. 1. After this research was completed we became aware of a number of related approaches and results. We learned from P. Deligne that results similar to ours are obtained in N. Katz's work $[\mathbf{K}]$ on rigid local systems by a more general and less elementary method. In particular, he sketched to us how one can obtain the classification of 5-dimensional braid representations from Katz's work (see also Remark 4 below).

2. We also learned of another approach using quiver theory (see [Ws] and, for a more general approach, $[\mathbf{S}]$ ). The following result appears explicitly in a preprint by Westbury:

Recall that in the $P S L(2, \mathbf{Z})$-quotient of $B_{3}$ the elements $A B A$ and $A B$ have order 2 and 3 respectively. Let $n_{1}$ and $n_{2}$ be the dimensions of the eigenspaces of $A B A$, and let $m_{1}, m_{2}$ and $m_{3}$ be the dimensions of the eigenspaces of $A B$. Then there exists an indecomposable representation of $\operatorname{PSL}(2, \mathbf{Z})$ if and only if $n_{i} \geq m_{j}$ for $i=1,2$ and $j=1,2,3$. Moreover, the parameter space for representations of this type has dimension $d^{2}-n_{1}^{2}-n_{2}^{2}-$ $m_{1}^{2}-m_{2}^{2}-m_{3}^{2}+1$. This result is in accordance with our findings for $d \leq 5$, but does not say anything about when the representations are simple (e.g. for $d=4$ there also exist indecomposable but not simple representations with $A B$ only having 2 eigenvalues).

3. We do not expect our methods to work for dimension $\geq 6$ without significant changes. For one, we can not expect ordered triangular forms for $A$ and $B$ : Indeed, if this were the case, $\operatorname{Tr}(A B A)=0$ for any simple 6-dimensional representation of $B_{3}$, by Prop. 1.3(a), i.e., $n_{1}=n_{2}=3$. To get a counterexample, it suffices to find $6 \times 6$ matrices of order 2 and 3 which generate the whole ring of $6 \times 6$ matrices and such that $n_{1}=4$ and $n_{2}=2$.

Similarly, the result quoted under 2 also shows that we will have more parameters than the eigenvalues for $d \geq 6$ and suitable values of $m_{1}, m_{2}, m_{3}$, $n_{1}, n_{2}$.

4. There exists a beautiful and simple argument which considerably narrows down which polynomials can occur in the simplicity statement of Theorem 2.9, and which also explains the nature of the factors in the polynomials $Q_{i j}^{(d)}$ to some extent. We were told this argument by P. Deligne:

Let $V$ be a $d$-dimensional $B_{3}$ module on which the central element $\zeta=$ $\left(\sigma_{1} \sigma_{2}\right)^{3}$ acts as a scalar $\delta$. Assume that $V$ has an $r$-dimensional $B_{3}$-submodule $W$ on which $\sigma_{1}$ acts with eigenvalues $\lambda_{1}, \ldots, \lambda_{r}$. Comparing 
determinants of the restriction of $\zeta$ to $W$, we get that

$$
\left(\lambda_{1} \ldots \lambda_{d}\right)^{6 r}=\left(\lambda_{1} \ldots \lambda_{r}\right)^{6 d} .
$$

Hence whenever $\zeta$ acts as a scalar and $(*)$ is NOT satisfied, the representation has to be simple. Unfortunately, this argument produces a sufficient but not a necessary condition for $V$ being simple.

5. A result which could be related to our findings is Coxeter's classification of finite quotients of $B_{3}$ defined by the additional relation $\sigma_{i}^{p}=1$, for $i=1,2$ : The additional relation defines a finite quotient if and only if $p \leq 5$ (see $[\mathbf{C}]$ ).

\section{An Application to Tensor Categories.}

3.1. We shall use the results of the previous section to compute categorical dimensions for certain simple objects in braided tensor categories related to exceptional Lie groups. We will not give much background information about tensor categories here (see e.g., $[\mathbf{D M}],[\mathbf{J S}],[\mathbf{K W}]$ or $[\mathbf{T}]$ ), as the application itself is rather elementary. The reader not familiar with the categorical language should think of the categories as representation categories of quantum groups.

Let $\mathcal{C}$ be a semisimple ribbon tensor category with trivial object $\mathbb{1}$ over an algebraically closed field $K$ (see [T] for precise definitions); this means, in particular, that End $(X)$ is a semisimple $K$-algebra for any given object $X$ in $\mathcal{C}$, and the homomorphisms between 2 objects in $\mathcal{C}$ form a vector space over $K$.

In the following, let $Z$ be a simple selfdual object in $\mathcal{C}$; this means that the trivial object $\mathbb{1}$ appears with multiplicity one as a direct summand in $Z^{\otimes 2}$. Let $p \in \operatorname{End}\left(Z^{\otimes 2}\right)$ be the projection onto $\mathbb{1}$, and let $p_{1}=p \otimes 1$ and $p_{2}=1 \otimes p$ be elements in $\operatorname{End}\left(Z^{\otimes 3}\right)$. In the following we will always assume the following (rigidity) condition

$$
p_{2} p_{1} p_{2} \neq 0 \text {. }
$$

As $Z \otimes \mathbb{1}$ is canonically isomorphic to the simple object $Z, p_{2}(a \otimes 1) p_{2}$ is equal to a scalar multiple of $p_{2}$ for any $a \in \operatorname{End}\left(Z^{\otimes 2}\right)$. Let $Y$ be a direct summand in $Z^{\otimes 2}$, with respect to a chosen direct sum decomposition of $Z^{\otimes 2}$. This decomposition defines a projection $p_{Y}$ onto $Y$. We define the categorical dimension $\operatorname{dim} Y$ by

$$
(\operatorname{dim} Y) p_{2}=(\operatorname{dim} Z)^{2} p_{2}\left(p_{Y} \otimes 1\right) p_{2} .
$$

This definition depends on a choice of $\operatorname{dim} Z$. In the following we will always require $\operatorname{dim} \mathbb{1}=1$. Taking for $p_{Y}$ the projection $p_{1}$, it follows from (3.1) and (3.2) that $\operatorname{dim} Z$ is uniquely determined up to a $\operatorname{sign} ; \operatorname{dim} Y$ is independent of the choice of the sign. So, in particular, if $Z^{\otimes 2}$ contains a subobject isomorphic to $Z, \operatorname{dim} Z$ is uniquely determined by (3.2). It is not hard to 
check that for subobjects of $Z^{\otimes 2}$ this definition coincides with the usual definition of categorical trace as e.g., for ribbon tensor categories (see $[\mathbf{T}]$ ).

3.2. Assume that $\mathcal{C}$ allows a braiding (see e.g., $[\mathbf{J S}],[\mathbf{T}]$ ). For the purpose of this article, it suffices to know that this entails that there exists a canonical endomorphism $c \in \operatorname{End}\left(Z^{\otimes 2}\right)$ such that $c_{1} c_{2} c_{1}=c_{2} c_{1} c_{2}$, with $c_{i}$ elements in $\operatorname{End}\left(Z^{\otimes 3}\right)$ defined as before the $p_{i}$. We have the following simple:

Lemma. Assume that $Z^{\otimes 2}$ decomposes as a direct sum $\bigoplus_{i} Y_{i}$ of $d$ mutually nonisomorphic simple objects $Y_{i}$, each of which has nonzero dimension. Moreover, we assume that $c$ acts on $Y_{i}$ via the scalar $\lambda_{i}$, and $\lambda_{i} \neq \lambda_{j}$ for $i \neq j$.

Then $\operatorname{Hom}\left(Z, Z^{\otimes 3}\right)$ is a simple d-dimensional $B_{3}$-module, with the action defined via $\sigma_{j} f=c_{j} \circ f$ for all $f \in \operatorname{Hom}\left(Z, Z^{\otimes 3}\right)$ and $j=1,2$; moreover, each eigenvalue of $c_{j}$ has multiplicity 1.

Proof. Let $p^{(i)} \in \operatorname{End}\left(Z^{\otimes 2}\right)$ be the projection onto $Y_{i}$, with $p=p^{(1)}$ being the projection onto $\mathbb{1} \subset Z^{\otimes 2}$ and let $\iota: \mathbb{1} \rightarrow Z^{\otimes 2}$ be a nonzero homomorphism. It follows from rigidity that $\left(p^{(i)} \otimes 1\right) \circ(1 \otimes \iota)$ is a nonzero homomorphism and that $\operatorname{dim} \operatorname{Hom}\left(Z, Z^{\otimes 3}\right)=\operatorname{dim} \operatorname{End}\left(Z^{\otimes 2}\right)=d$; it can also be checked explicitly for the examples below. Hence $V=\operatorname{Hom}\left(Z, Z^{\otimes 3}\right)$ is a $d$-dimensional vector space on which both $c_{1}$ and $c_{2}$ act via concatenation of morphisms. Moreover, it has a basis of eigenvectors $\left(p^{(i)} \otimes 1\right) \circ(1 \otimes \iota)$ of $c_{1}$. If this representation of $B_{3}$ were not simple, fix a composition series of $V$, and pick an eigenprojection $p^{(i)}$ of $c$ such that the eigenprojections $p^{(i)} \otimes 1$ and $p_{1}=p \otimes 1$ of $c_{1}$ act nonzero on different simple factors. As $p_{1}$ is conjugate to $p_{2}=1 \otimes p$ (as $c_{1}$ and $c_{2}$ are conjugate), we can conclude $p_{2}\left(p^{(i)} \otimes 1\right) p_{2}=0$, which would contradict the assumption about nonzero dimensions.

Corollary. Using the notations of the Lemma, with $Y_{1} \cong \mathbb{1}$, we have

$$
\operatorname{dim} Y_{i}=\frac{Q_{1 i}^{(d)}(\operatorname{dim} Z)^{2}}{P_{1}^{(d)}\left(\lambda_{1}\right) P_{i}^{(d)}\left(\lambda_{i}\right)}
$$

where $Q_{1 i}, P_{1}^{(d)}\left(\lambda_{1}\right)$ and $P_{i}^{(d)}\left(\lambda_{i}\right)$ are as in Section 2.8 .

Proof. Observe that the projection onto the eigenspace of $A$ with eigenvalue $\lambda$ is given by $P_{i}^{(d)}(A) / P_{i}^{(d)}\left(\lambda_{i}\right)$ The claim now follows from (3.2) and Prop. $2.8(\mathrm{a})$. 
3.3. Let us first consider the case with $\mathcal{C}$ a braided tensor category whose Grothendieck semiring is isomorphic to the semiring of the representation category of the Lie group $G$ with $G$ being an orthogonal group $O(N)$ or a symplectic group $S p(N)$. We take as object $Z$ the vector representation of $G$. It is well-known that in this case $Z \otimes Z \cong \mathbb{1} \oplus X \oplus Y$, with $X$ and $Y$ simple objects corresponding to the antisymmetrization and a subrepresentation of the symmetrization (traceless tensors) of the 2 nd tensor power of the vector representation. It can be shown that in this case (only using the assumptions of braiding, and the given Grothendieck semi-ring), the eigenvalues are of the form $\alpha q,-\alpha q^{-1}, \alpha r^{-1}$, with $\alpha$ a 4-th root of unity and $q, r \in K$. Moreover, $r$ is \pm a power of $q$, depending on the given category (see below). Using the notations $[n]=q^{n}-q^{-n}$ and $[\lambda+n]=r q^{n}-r^{-1} q^{-n}$, it follows from Corollary 3.2 that

$$
\operatorname{dim} Z=\alpha^{2}\left(\frac{[\lambda]}{[1]}+1\right)=\alpha^{2}\left(\frac{r-r^{-1}}{q-q^{-1}}+1\right),
$$

and

$$
\operatorname{dim} X=\frac{[\lambda-1]+[2]}{[2]} \frac{[\lambda]}{[1]}, \quad \operatorname{dim} Y=\frac{[\lambda+1]+[2]}{[2]} \frac{[\lambda]}{[1]}
$$

This method can be extended to define similar functions also for objects in higher tensor powers of $Z$, which are labeled by Young diagrams (see $[\mathbf{W n}$, Theorem 5.5]). The $q$-dimensions for $G=O(N)$ are obtained by setting $\lambda=N-1$, for $G=S p(N)$ they are obtained by setting $\lambda=-N-1$. More generally, it is possible to reconstruct such categories similarly as it was done for categories of type $\mathrm{A}$ in $[\mathbf{K W}]$; however, in this case we would need the assumption of the category being braided. So in this comparatively simple case knowledge about braid representations allows us to reconstruct the tensor category.

3.4. The discussion in 3.3 can be carried over to exceptional Lie groups to some (so far rather limited) extent. We use the notations $[n]=s^{n / 2}-s^{-n / 2}$ and $[\lambda+n]=t^{1 / 2} s^{n / 2}-t^{-1 / 2} s^{-n / 2}$ for $n \in \mathbf{N}$ and $\lambda$ a formal variable; in our formulas, the result will be independent of the choice of square roots of $s$ and $t$. It can be shown that for an exceptional Lie algebra $\mathfrak{g}$, the 2 nd tensor product $\mathfrak{g}^{\otimes 2}$ of its adjoint representation decomposes as a direct sum $\mathbb{1} \oplus \mathfrak{g} \oplus X_{2} \oplus Y_{2} \oplus Y_{2}^{*}$. Here we use the same notations as in [De]. We are going to use the following results about quantum groups, which are essentially due to Drinfeld [Dr2] (for a description of the relationship between operators $c$ (braiding operators) and quantum Casimir (= twist) also see e.g., [T]); here $a_{Y}$ denotes the scalar via which the Casimir acts on a simple $\mathfrak{g}$-module $Y$ : 
(i) The braiding operator $c$ acts on the simple summand $Y \subset \mathfrak{g}^{\otimes 2}$ via the scalar $\pm q^{a_{Y}-2 a_{\mathfrak{g}}}$, with the sign depending on whether $Y$ is in the symmetrization or antisymmetrization of $\mathfrak{g}^{\otimes 2}$,

(ii) the operator $\left(c_{1} c_{2}\right)^{3}$ acts on the simple summand $K \subset \mathfrak{g}^{\otimes 3}$ via the scalar $q^{2 a_{K}-6 a_{\mathfrak{g}}}$.

Now setting $q^{2 a_{\mathfrak{g}}}=s^{-3}$ and $q^{a_{Y_{2}}-2 a_{\mathfrak{g}}}=t$, and using the formulas for the action of the Casimir in [De], one sees easily that the eigenvalues of $c$ (acting on $\mathfrak{g}^{\otimes 2}$ ) are $s^{6}$ (for $\mathbb{1}$ ), $-s^{3}$ (for $\left.\mathfrak{g}\right),-1$ (for $X_{2}$ ), $t$ (for $Y_{2}$ ) and $s t^{-1}$ (for $Y_{2}^{*}$ ). It follows from this that $\operatorname{det}_{V}\left(c_{1}\right)=s^{10}$, where $\operatorname{det}_{V}\left(c_{1}\right)$ is the determinant of the linear operator via which $c_{1}$ acts on $V$.

In order to completely determine the 5-dimensional representation of $B_{3}$ on $V=\operatorname{Hom}\left(\mathfrak{g}, \mathfrak{g}^{\otimes 3}\right)$, we only need to compute the scalar $\delta$ by which the central element $\left(c_{1} c_{2}\right)^{3}$ acts on $V$. This is equivalent to determining the 5-th root $\gamma$ of $\operatorname{det}_{V}\left(c_{1}\right)$ given by $\delta=\gamma^{6}=\operatorname{det}_{V}\left(c_{1}\right) \gamma=s^{10} \gamma$. By (ii), we get $\delta=q^{2 a_{\mathfrak{g}}-6 a_{\mathfrak{g}}}=s^{12}$. Combining the last 2 formulas, we get $\gamma=s^{2}$. One can now check, using Corollary 3.2, that

$$
\begin{aligned}
\operatorname{dim} \mathfrak{g} & =\frac{[4][\lambda-6][\lambda+5]}{[2][\lambda-1][\lambda]} \\
\operatorname{dim} X_{2} & =\frac{[5][\lambda-6][\lambda+5][\lambda-4][\lambda+3][2 \lambda+4][2 \lambda-6]}{[1][\lambda][\lambda-1][\lambda+2][\lambda-3][2 \lambda][2 \lambda-2]}, \\
\operatorname{dim} Y_{2} & =\frac{[6][5][4][\lambda+5][\lambda-4][3 \lambda-6]}{[2][\lambda-1][\lambda][2 \lambda][2 \lambda-1][\lambda-2]} \\
\operatorname{dim} Y_{2}^{*} & =\frac{[6][5][4][\lambda-6][2 \lambda+6][3 \lambda+3][\lambda+2]}{[2][\lambda-1][\lambda][2 \lambda-2][2 \lambda-1][2 \lambda+4][\lambda+1]}
\end{aligned}
$$

These rational functions are $s$-deformations of formulas given in $[\mathbf{D e}]$ for the classical case.

3.5. The computations above in the orthogonal case were used by Toledano Laredo in $[\mathbf{T L}]$ in the context of fusion of representations of loop groups, based on unpublished notes of the second named author. We have been informed by Antony Wassermann that he uses our results for 5-dimensional braid representations in connection with fusion of representations of loop groups corresponding to exceptional Lie groups.

\section{References}

[BLM] J.S Birman, D.D. Long and J.A. Moody, Finite-dimensional representations of Artin's braid group, Contemp. Math., 169, Amer. Math. Soc., Providence, RI, (1994), 123-132. 
[C] H.S.M. Coxeter, Factor Groups of the Braid Group, Proceedings of the Fourth Can. Math. Congress, Banff 1957, University of Toronto Press, (1959), 95-122.

[De] P. Deligne, La série exceptionnelle de groupes de Lie, C. R. Acad. Sci. Paris Sér. I Math., 322(4) (1996), 321-326.

[DM] P. Deligne and J. Milne, Tannakian Categories, Lecture Notes in Mathematics, 900, Springer Verlag.

[Dr1] V. Drinfeld, Quantum Groups, Proceedings for the ICM Berkeley, (1986), 798-820.

[Dr2] _ On almost cocommutative Hopf algebras, Leningrad Math. J., 1 (1990), 321-243.

[JS] A. Joyal and R. Street, The geometry of tensor calculus, Adv. Math., 88 (1991).

$[\mathrm{K}] \quad$ N. Katz, Rigid Local Systems, Annals of Mathematics Studies, 139, Princeton University Press.

[KW] D. Kazhdan and H. Wenzl, Reconstructing monoidal categories, Advances in Soviet Math., 16(2) (1993), 111-136.

[S] A. Schofield, General representations of quivers. Proc. London Math. Soc. (3), 65(1) (1992), 46-64.

[TL] V. Toledano Laredo, Fusion of Positive Energy Representations of $\operatorname{LSpin}_{2 n}$, Thesis, University of Cambridge, 1997.

[T] V.G. Turaev, Quantum Invariants of Knots and 3-Manifolds, de Gruyter (1994).

[V] P. Vogel, Algebraic structures on modules of diagrams, preprint.

[Wa] A. Wassermann, Operator algebras and conformal field theory III, Invent. Math., (1998).

[Wn] H. Wenzl, Quantum groups and subfactors of type B, $C$ and D, Comm. Math. Phys., 133 (1990), 383-432.

[Ws] B. Westbury, On the character varieties of the modular group, preprint, University of Nottingham, 1995.

Received June 1, 1999. The second author was supported in part by NSF grant \# DMS 97-06839.

University of California, San Diego

LA Jolla, CA 92093-0112

E-mail address: ituba@euclid.ucsd.edu

University of California, San Diego

LA Jolla, CA 92093-0112

E-mail address: wenzl@brauer.ucsd.edu 